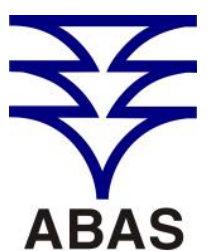

ASSOCIACAO BRASLERRADE
AGUAS SUBTERRANEAS www.abas.org

\section{AVALIAÇÃO DA VULNERABILIDADE DE AQUÍFEROS LOCALIZADOS NA REGIÃO CENTRAL DE CANOAS - RS}

\author{
EVALUATION OF AQUIFERS VULNERABILITY LOCALIZED IN THE \\ CENTRAL REGION OF CANOAS - RS
}

\author{
Guilherme Lahm Feron ${ }^{1}$, Pedro Antonio Roehe Reginato ${ }^{2}$
}

Artigo recebido em: 28/02/2014 e aceito para publicação em: 30/05/2014.

\begin{abstract}
This paper presents the study of natural vulnerability of aquifers localized in the central region of Canoas city (metropolitan region of Porto Alegre, RS), based in the GOD method. For the study were analyzed geological and hidrogeological data from 46 wells, obtained at the SIAGAS data base (CPRM). The main aquifers of that region are associated with shales which have thickness between $6 \mathrm{~m}$ and $50 \mathrm{~m}$ and are located at depths between $30 \mathrm{~m}$ and $175 \mathrm{~m}$ and, with sandstones that possesses medium thickness of $8,2 \mathrm{~m}$ and medium depths of $155,5 \mathrm{~m}$. For the $\mathrm{G}$ parameter were defined values between 0,2 (confined) to 0,6 (unconfined covered), the $\mathrm{O}$ parameter received values between 0,4 and 0,7 (because the main cover strata in the region being clays, silts and sands) and the D parameter, corresponding to the depths of static level stayed between 0,6 and 0,9 . The aquifers presented three vulnerability classes (negligible, low and medium), being the biggest area covered by low vulnerability $(94,12 \%)$. Despite of the aquifers of the study region present low natural vulnerability to contamination, the lack of control and the inadequate installation of those wells may cause the contamination of groundwater, thus, the low vulnerability by itself it's not an guarantee of non contamination for the aquifer.
\end{abstract}

Keywords: Natural Vulnerability. GOD Method. Canoas-RS.

Resumo: Este artigo apresenta o estudo da vulnerabilidade natural dos aquíferos que estão localizados na região central do município de Canoas (região metropolitana de Porto Alegre, RS), com base na utilização do método GOD. Para o estudo foram analisados dados geológicos e hidrogeológicos de 46 poços tubulares obtidos junto ao banco de dados SIAGAS (CPRM). Os principais aquíferos existentes na região estão associados a folhelhos que apresentam espessuras entre $6 \mathrm{~m}$ e $50 \mathrm{~m}$ e estão localizados em profundidades entre 30 e $175 \mathrm{~m}$ e, a arenitos que possuem espessura média de $8,2 \mathrm{~m}$ e profundidade média de $155,5 \mathrm{~m}$. Para o parâmetro G foram definidos valores entre 0,2 (confinado) e 0,6 (não confinado coberto), o parâmetro $\mathrm{O}$ recebeu valores entre 0,4 e 0,7 (devido aos principais estratos de cobertura na região serem argilas, siltes e areias) e o parâmetro $\mathrm{D}$, correspondente à profundidade do nível estático ficou entre 0,6 e 0,9 . Os aquíferos apresentam três classes de vulnerabilidade (insignificante, baixa e média), sendo a maior área, abrangida por baixa vulnerabilidade $(94,12 \%)$. Apesar de os aquíferos da região de estudo apresentarem baixa vulnerabilidade natural à contaminação, a falta de controle e a instalação inadequada de poços tubulares pode causar a contaminação da água subterrânea, e sendo assim, a baixa vulnerabilidade por si só não é garantia da não contaminação do aquífero.

Palavras-chave: Vulnerabilidade Natural. Método GOD. Canoas-RS.

\title{
INTRODUÇÃO
}

As águas subterrâneas são, geralmente, fontes de águas limpas e de boa qualidade. No Brasil, atualmente, 39\% dos municípios são abastecidos exclusivamente através de poços tubulares que captam essas fontes. No Rio Grande do Sul, 59\% dos municípios do Estado são abastecidos totalmente por águas subterrâneas e $13 \%$ dos mu- nicípios utilizam a água subterrânea como complemento para o abastecimento (ANA, 2010).

No município de Canoas, localizado na região metropolitana de Porto Alegre, existem 115 poços tubulares registrados no banco de dados do Sistema de Informações de Água Subterrânea (SIAGAS, 2013) do Serviço Geológico do

\footnotetext{
${ }^{1}$ Mestrando do Programa de Pós-Graduação em Engenharia de Minas, Metalúrgica e dos Materiais da UFRGS (guiferon@gmail.com).

2 Professor do Departamento de Hidromecânica e Hidrologia do Instituto de Pesquisas Hidráulicas (IPH) da Universidade Federal do Rio Grande do Sul (pedro.reginato@ufrgs.br).
} 
Brasil (Companhia de Recursos Minerais CPRM). Esses poços são utilizados por diversos empreendimentos como: indústrias, condomínios residenciais e empresas particulares. Porém, em função do grande numero de poços instalados e da falta de monitoramento, alguns poços podem se tornar vias de contaminação da água subterrânea, comprometendo, assim, a qualidade da água presente no aquífero.

A contaminação do aquífero depende de diversos fatores, sendo que os principais estão relacionados com as características dos estratos confinantes, com a capacidade de atenuação dos contaminantes pelo meio e com a acessibilidade do contaminante a água subterrânea (FOSTER et. al., 2006; MARQUEZAN, 2008). A análise e a integração desses fatores pode ser utilizada na avaliação da vulnerabilidade de aquíferos.

A preocupação com a vulnerabilidade natural dos aquíferos localizados no município de Canoas levou ao desenvolvimento deste trabalho que tem como objetivo principal promover a ca-

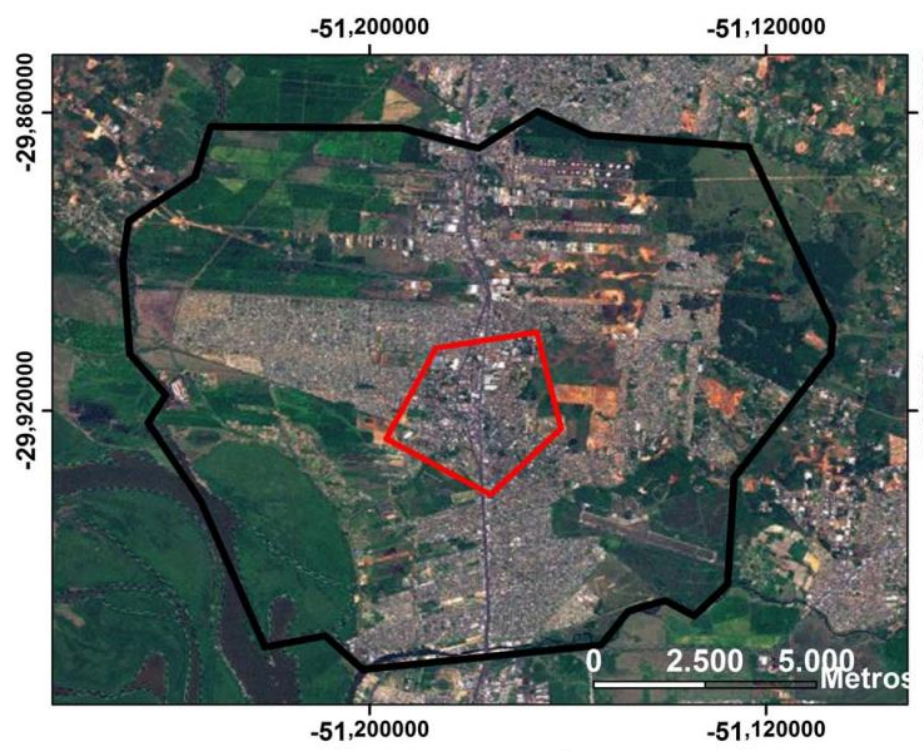

Mapa de Localização da Área de Estudo

\section{Legenda}

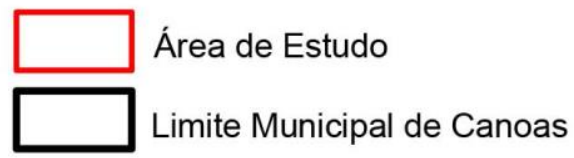

Figura 1 - Localização da área de estudo Figure 1- Location of Study area

De acordo com a Carta Geológica do Estado do Rio Grande do Sul (CPRM, 2008), os principais depósitos sedimentares aflorantes no município, estão representados pelos Depósitos racterização dos aquíferos captados por poços tubulares localizados na região central do município de Canoas e avaliar a vulnerabilidade natural desses aquíferos. A avaliação da vulnerabilidade foi realizada com o emprego da metodologia GOD ("Groundwater Polluition Risk Evaluation", Foster e Hirata, 1988; Foster et. al. 2006).

\section{LOCALIZAÇÃO, CARACTERIZAÇÃO GE- OLÓGICA E HIDROGEOLÓGICA DA ÁREA}

A área de estudo está localizada na parte central do município de Canoas, na região metropolitana de Porto Alegre (Figura 1), cujas coordenadas são $29^{\circ} 54^{\prime} 36^{\prime \prime}$ Sul e $51^{\circ} 10^{\prime} 48^{\prime \prime}$ Oeste. O município possui divisa com os municípios de Esteio, Cachoeirinha, Nova Santa Rita e a capital, Porto Alegre. A altitude mínima do município é de $4 \mathrm{~m}$, a máxima fica em torno de $33 \mathrm{~m}$ sendo que a média é de $8 \mathrm{~m}$ acima do nível do mar (IBGE, 2011; FAMURS, 2013).
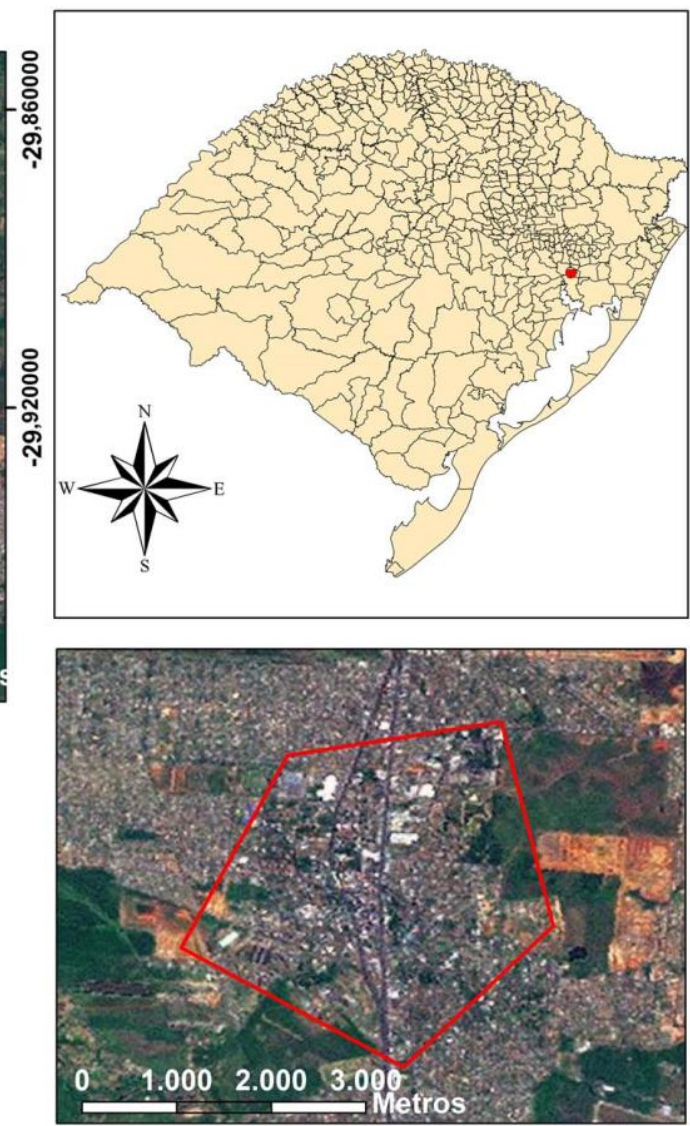


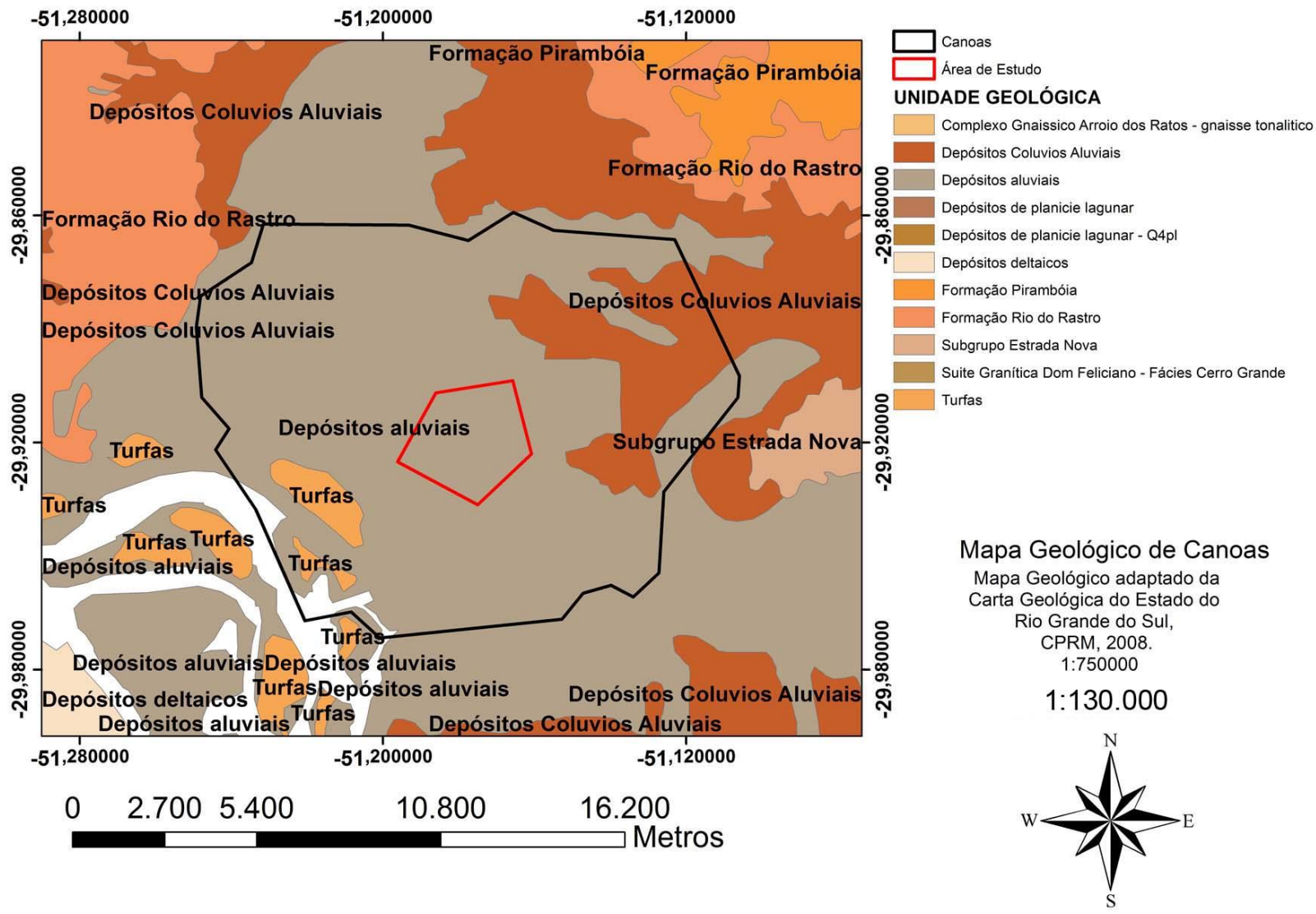

Figura 2 - Mapa Geológico de Canoas (adaptado de CPRM, 2008)

Figure 2 - Canoas Geologic Map (adapted from CPRM, 2008)

Segundo Viero (2010) a área de estudo está inserida na Depressão Gaúcha e se localiza na Depressão do Rio Jacuí, sendo que as litologias predominantes estão representadas por arenitos, folhelhos, siltitos e argilitos das Formações Rio Bonito, Palermo, Irati e Rio do Rasto e os arenitos e conglomerados das Formações Piramboia e Sanga do Cabral. Essas litologias ocorrem em diferentes profundidades e são recobertas pelos depósitos aluviais e colúvio-aluviais.

Conforme Machado e Freitas (2005) e Viero (2010) no município de Canoas há ocorrência de duas unidades hidrogeológicas denominadas de Sistema Aquífero Quaternário Costeiro II e Aquitardos Permianos (Figura 3).

O Sistema Aquífero Quaternário Costeiro II é formado por diferentes aquíferos que estão associados aos depósitos sedimentares (camadas de areias finas inconsolidadas e esbranquiçadas intercaladas por camadas de argilas de coloração cinza). As capacidades específicas variam de baixas a médias, entre 0,5 e $1,5 \mathrm{~m} / \mathrm{h} / \mathrm{m}$ e os sólidos totais dissolvidos variam entre 600 e $2000 \mathrm{mg} / \mathrm{l}$.

Os Aquitardos Permianos, segundo Machado e Freitas (2005) e Viero (2010) estão associados a rochas do tipo siltitos argilosos, argilitos, folhelhos e pequenas camadas de margas e arenitos. Quando essas rochas apresentam fraturas há possibilidade de formação de aquíferos fraturados, que foram considerados pelos autores como limitados e de baixa capacidade de produção. Nesse caso, os aquíferos apresentam capacidades específicas baixas, inferiores a $0,1 \mathrm{~m}^{3} / \mathrm{h} / \mathrm{m}$ e as águas podem ser duras com grandes quantidades de cálcio e magnésio. Segundo Marquezan (2008) a formação dos aquíferos fraturados está associada as intrusões de diabásios que cortam os siltitos e folhelhos, gerando um sistema de faturamento que dá origem a esse tipo de aquífero. 


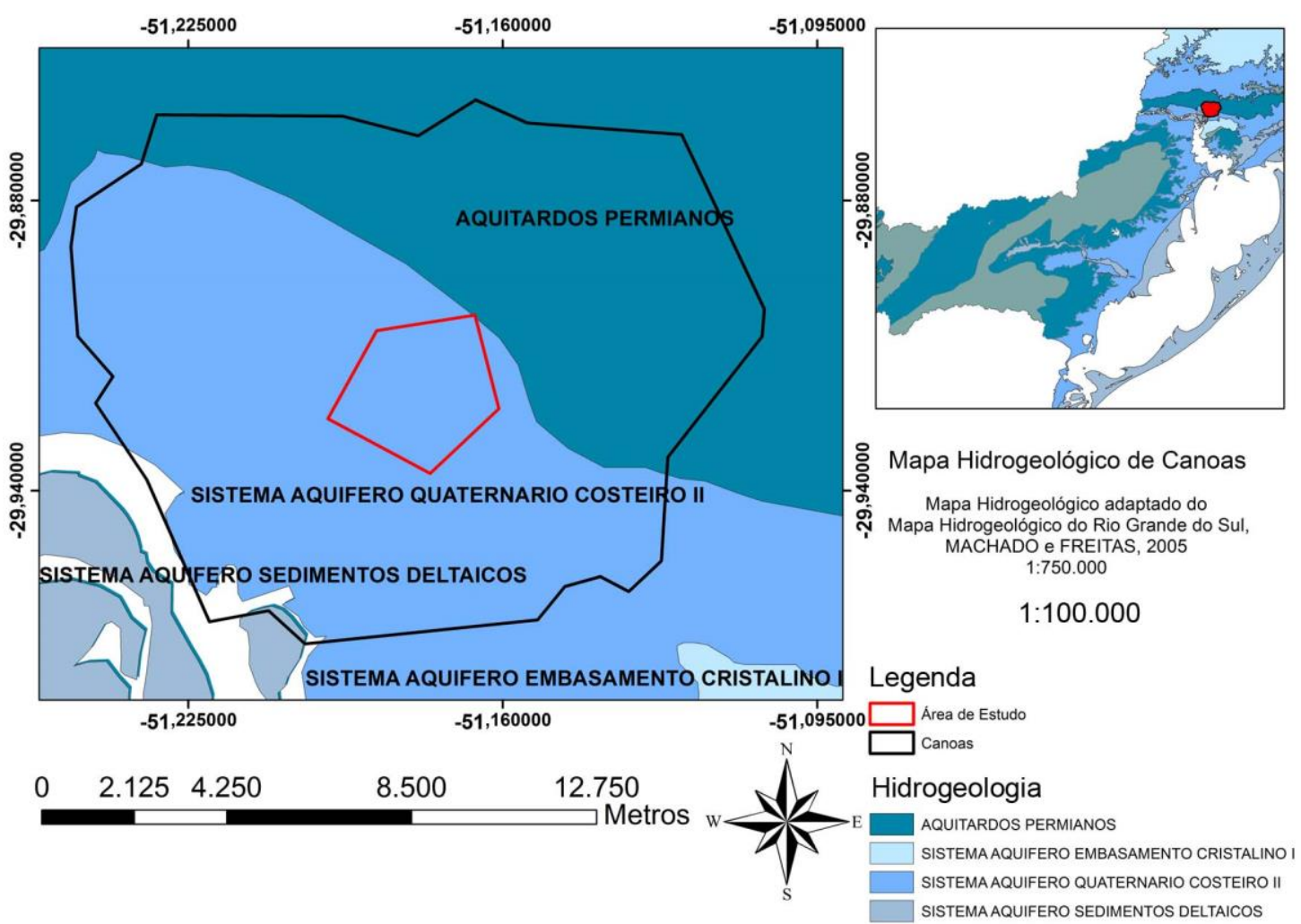

Figura 3 - Mapa Hidrogeológico de Canoas (adaptado de Machado e Freitas, 2005)

Figure 3 - Canoas Hidrogeologic Map (adapted from Machado e Freitas, 2005)

\section{MATERIAIS E MÉTODOS}

A caracterização hidrogeológica da área de estudo foi feita com base na avaliação de dados geológicos e hidrogeológicos disponíveis em publicações e na interpretação dos perfis geológicoconstrutivos dos poços tubulares identificados na região.

A identificação dos poços tubulares foi realizada através da consulta ao banco de dados do Sistema de Informações de Água Subterrânea (SIAGAS, 2013), sendo que as principais informações analisadas foram: nível estático, perfil geológico, profundidade das entradas de água, tipo de uso previsto e o proprietário, vazão após a estabilização, cota do terreno e localização do poço.

Foram encontrados, ao total, 115 pontos de captação no SIAGAS para o município de Canoas sendo que na zona central há uma maior concentração de poços tubulares (46) que são utilizados, na sua maioria, por condomínios residenciais. Em função disso, essa região foi selecionada para o estudo da vulnerabilidade.

A avaliação da vulnerabilidade dos aquíferos foi feita com base na aplicação da metodologia GOD (Foster e Hirata, 1988; Foster et. al. 2006). Como a área de estudo consiste numa zona altamente urbanizada e relativamente pequena, a utilização de outros métodos, como o DRASTIC (Aller et. al. 1987), se tornou inviável. Os parâmetros do método GOD foram identificados levando em conta os dados geológicos e hidrogeológicos contidos nos relatórios técnicos e perfis construtivos dos poços retirados do SIAGAS. A definição dos valores para cada parâmetro foi baseada na interpretação desses dados e na comparação com os valores padrões definidos pela metodologia (Figura 4).

O parâmetro G, ou Grau de Confinamento do Aquífero, foi classificado através da identificação do estrato aquífero aonde se encontra a primeira entrada d'água de cada poço. Esta escolha foi tomada devido à existência de diversas camadas aquíferas na região, assim, optou-se por adotar o aquífero mais raso presente em cada perfil. Os poços que não continham dados da profundidade das entradas de água ou não possuíam perfil construtivo não foram utilizados. Em função disso, 3 poços (P033, P076 e P081) foram descartados. Com os valores selecionados para todos os poços foi gerado um mapa de classificação do parâmetro $G$, elaborado a partir da interpolação dos valores atribuídos para cada poço e utilizando a ferramenta Interpolate do ArcGis 10 (ESRI, 2010). 


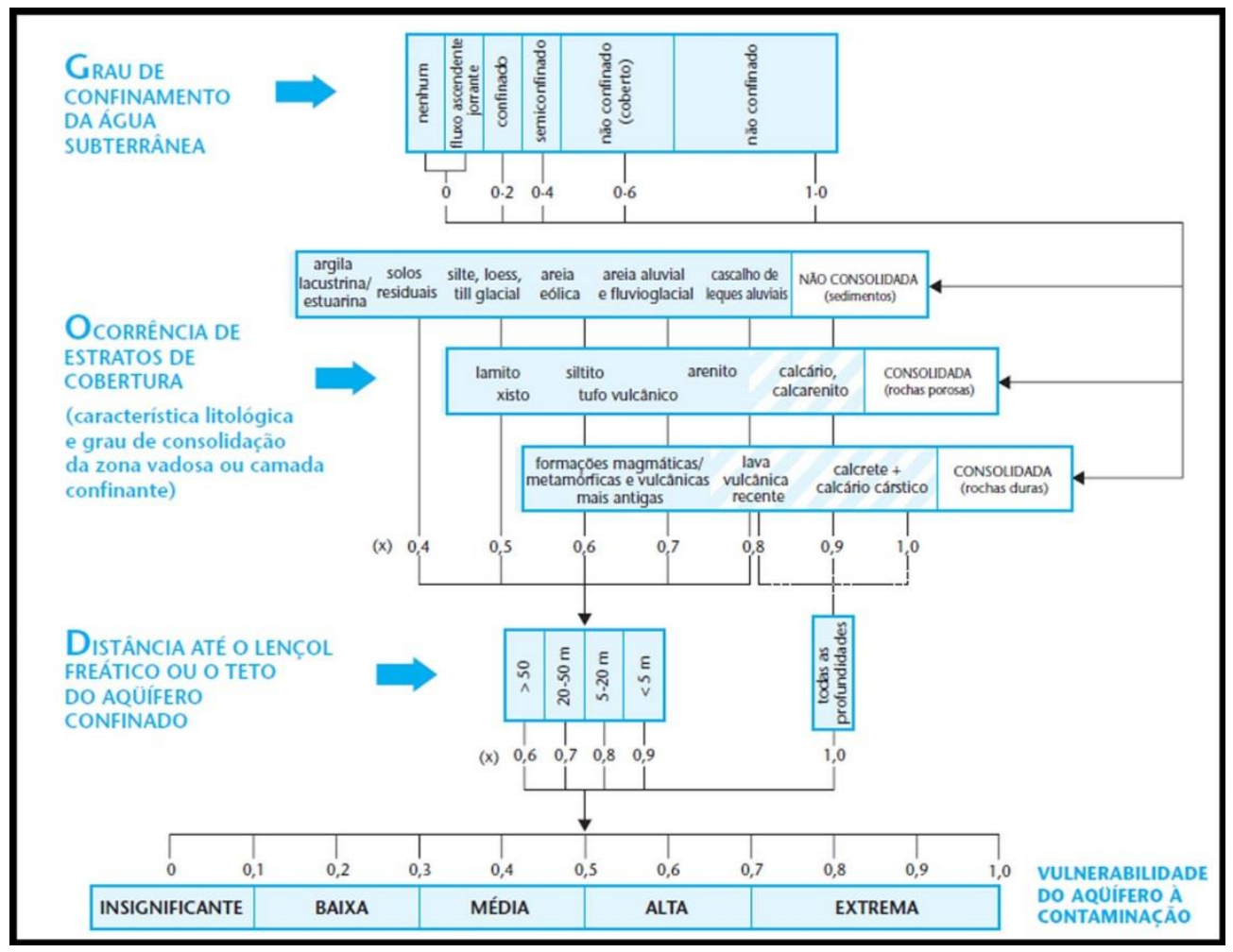

Figura 4 - Descrição dos Parâmetros e Valores Orientadores para aplicação da Metodologia GOD (FOSTER e HIRATA, 1988; FOSTER et. al. 2006)

Figure 4 - Description of Parameters and Guiding Values for applying the GOD methodology (Foster and HIRATA, 1988, FOSTER et al 2006)

O parâmetro $\mathrm{O}$, ou Ocorrência de Estratos de Cobertura, foi classificado identificando-se as camadas da zona não saturada logo acima do nível estático de cada poço e adotando-se os valores sugeridos pelo método. Os poços que não possuíam perfil geológico não foram utilizados ( $\mathrm{P} 033$, P076 e P081). A partir dos valores atribuídos para cada poço foi gerado um mapa com a interpolação dos pontos.

O parâmetro D, Distância ou Profundidade até o Nível de Água foi classificado, para cada poço, conforme indicado no método GOD e interpolado para todos os poços da área. A classificação adotada foi de 0,9 para profundidades de $0 \mathrm{~m}$ a $5 \mathrm{~m} ; 0,8$ para valores entre $5 \mathrm{~m}$ e $20 \mathrm{~m} ; 0,7$ para as variações de $20 \mathrm{~m}$ a $50 \mathrm{~m}$ e índice 0,6 para valores de profundidade de nível estático maiores que $50 \mathrm{~m}$. Um poço foi descartado pois não tinha informação sobre o nível estático (P097).

O Mapa de Vulnerabilidade foi obtido através da ferramenta Raster Calculator do ArcGis 10 (ESRI, 2010). Foi executada a multiplicação dos dados no formato raster, pixel a pixel, para gerar uma nova imagem, onde cada pixel tem um valor atribuído igual ao resultado da multiplicação dos valores das imagens originais (MEDEIROS et. al., 2011). Os mapas dos parâmetros $\mathrm{G}, \mathrm{O}$ e $\mathrm{D}$ foram multiplicados e o resultado gerado foi o Mapa de Vulnerabilidade Natural GOD.

\section{RESULTADOS E DISCUSSÃO}

$\mathrm{Na}$ área de estudo foram selecionados 46 poços tubulares (Figura 5) cujos dados (identificação, coordenadas, nível estático, uso da água, cota, presença de perfil litológico) são apresentados na Tabela 1.

Os dados geológicos dos poços tubulares foram interpretados e utilizados na elaboração de seções geológicas (Figura 5) que permitiram avaliar a distribuição estratigráfica, bem como características hidrogeológicas dos aquíferos (tipo de aquífero, profundidade de ocorrência, espessura). Nas figuras 6 e 7 são apresentadas duas seções geológicas que representam a distribuição estratigráfica das principais litologias identificadas na área. 
Tabela 1 - Identificação dos poços tubulares

Table 1 - Identification form of wells

\begin{tabular}{|c|c|c|c|c|c|c|}
\hline Identificação- № Poço & UTM (S) & UTM (E) & $\mathrm{NE}(\mathrm{m})$ & Uso da água & CT (m) & Perfil Litológico \\
\hline P008 & 6690702 & 482242 & 16,50 & Outros & 22 & $S$ \\
\hline P009 & 6689513 & 481247 & 7,00 & Industrial & 8 & $S$ \\
\hline P010 & 6690666 & 483672 & 32,87 & Outros & 20 & S \\
\hline P011 & 6689276 & 482353 & 20,50 & Industrial & 12 & S \\
\hline P012 & 6690957 & 482268 & 43,50 & Condominio & 10 & $S$ \\
\hline P015 & 6690974 & 482375 & 16,80 & Condominio & 6 & $S$ \\
\hline P016 & 6689948 & 481938 & 40,72 & Condominio & 15 & $S$ \\
\hline P017 & 6689757 & 482126 & 20,37 & Condominio & 11 & $S$ \\
\hline P018 & 6689862 & 482177 & 13,90 & Condominio & 5 & $S$ \\
\hline P019 & 6689328 & 483185 & 4,27 & Condominio & 8 & $S$ \\
\hline P020 & 6689632 & 482626 & 13,46 & Condominio & 9 & $S$ \\
\hline P026 & 6690427 & 482377 & 20,80 & Outros & 26 & $S$ \\
\hline P027 & 6690356 & 482172 & 49,53 & Outros & 25 & $S$ \\
\hline P028 & 6690475 & 482295 & 11,84 & Outros & 24 & $S$ \\
\hline P030 & 6690370 & 482707 & 64,00 & Condominio & 21 & $S$ \\
\hline P033 & 6690837 & 482248 & 8,25 & Condominio & 17 & $\mathrm{~N}$ \\
\hline P035 & 6689776 & 484287 & 12,40 & Outros & - & $S$ \\
\hline P036 & 6688927 & 482945 & 9,00 & Condominio & - & $S$ \\
\hline P038 & 6691045 & 483497 & 21,00 & Outros & 30 & $S$ \\
\hline P039 & 6689323 & 483114 & 5,00 & Condominio & 19 & $S$ \\
\hline P040 & 6691575 & 483547 & 36,70 & Industrial & 28 & $S$ \\
\hline P044 & 6691265 & 483668 & 47,30 & Outros & 28 & $S$ \\
\hline P047 & 6690338 & 483770 & 11,00 & Condominio & 23 & $S$ \\
\hline P052 & 6691172 & 482715 & 31,34 & Condominio & 17 & $S$ \\
\hline P054 & 6690428 & 482270 & 14,00 & Condominio & 24 & $S$ \\
\hline P055 & 6691181 & 483440 & 38,86 & Industrial & 28 & $S$ \\
\hline P062 & 6691308 & 482355 & 10,77 & Condominio & 7 & $S$ \\
\hline P063 & 6691187 & 482656 & 28,56 & Condominio & 14 & $S$ \\
\hline P073 & 6690212 & 481676 & 4,40 & Industrial & 7 & $S$ \\
\hline P076 & 6690093 & 483123 & 40,30 & Condominio & 25 & $\mathrm{~N}$ \\
\hline P078 & 6691404 & 483289 & 46,70 & Industrial & 28 & $S$ \\
\hline P079 & 6690356 & 483152 & 52,21 & Condominio & 24 & $S$ \\
\hline P080 & 6690950 & 482264 & 22,50 & Condominio & 13 & $S$ \\
\hline P081 & 6691046 & 483202 & 42,00 & Industrial & 27 & $\mathrm{~N}$ \\
\hline P086 & 6690947 & 482796 & 43,35 & Outros & 26 & $S$ \\
\hline P088 & 6690783 & 481873 & 40,80 & Condominio & 24 & $S$ \\
\hline P090 & 6689481 & 483588 & 5,43 & Outros & - & $S$ \\
\hline P091 & 6690459 & 482595 & 30,00 & Outros & 31 & $S$ \\
\hline P092 & 6688386 & 483080 & 60.6 & Condominio & 10 & $S$ \\
\hline P095 & 6691705 & 483918 & 44,31 & Industrial & 28 & $S$ \\
\hline P097 & 6691063 & 483099 & - & Outros & 26 & $S$ \\
\hline P098 & 6691526 & 483232 & 57,10 & Industrial & 24 & $S$ \\
\hline P100 & 6691381 & 483573 & 28,00 & Industrial & 27 & $S$ \\
\hline P102 & 6691335 & 482990 & 35,00 & Industrial & 21 & $S$ \\
\hline P1811 & 6691415 & 482223 & 49,68 & Industrial & 5 & $S$ \\
\hline P2380 & 6689670 & 484298 & 10,80 & Outros & 19 & $S$ \\
\hline
\end{tabular}

$\mathrm{NE}=$ nível estático; $\mathrm{CT}=$ cota do terreno. 


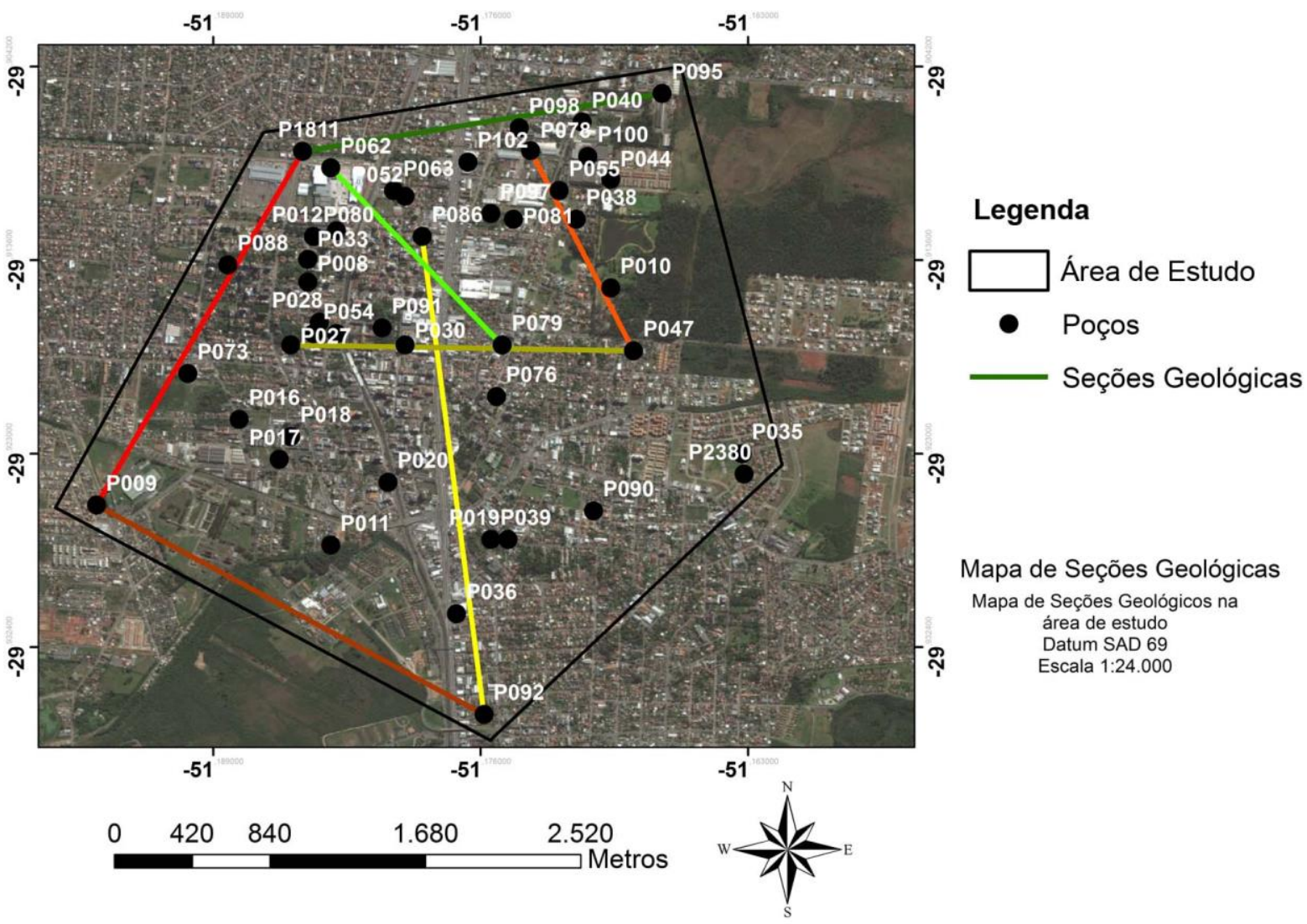

Figura 5 - Distribuição dos Poços Tubulares e das Seções Geológicas Figure 5 - Distribution of Tubular Wells and Geological Sections

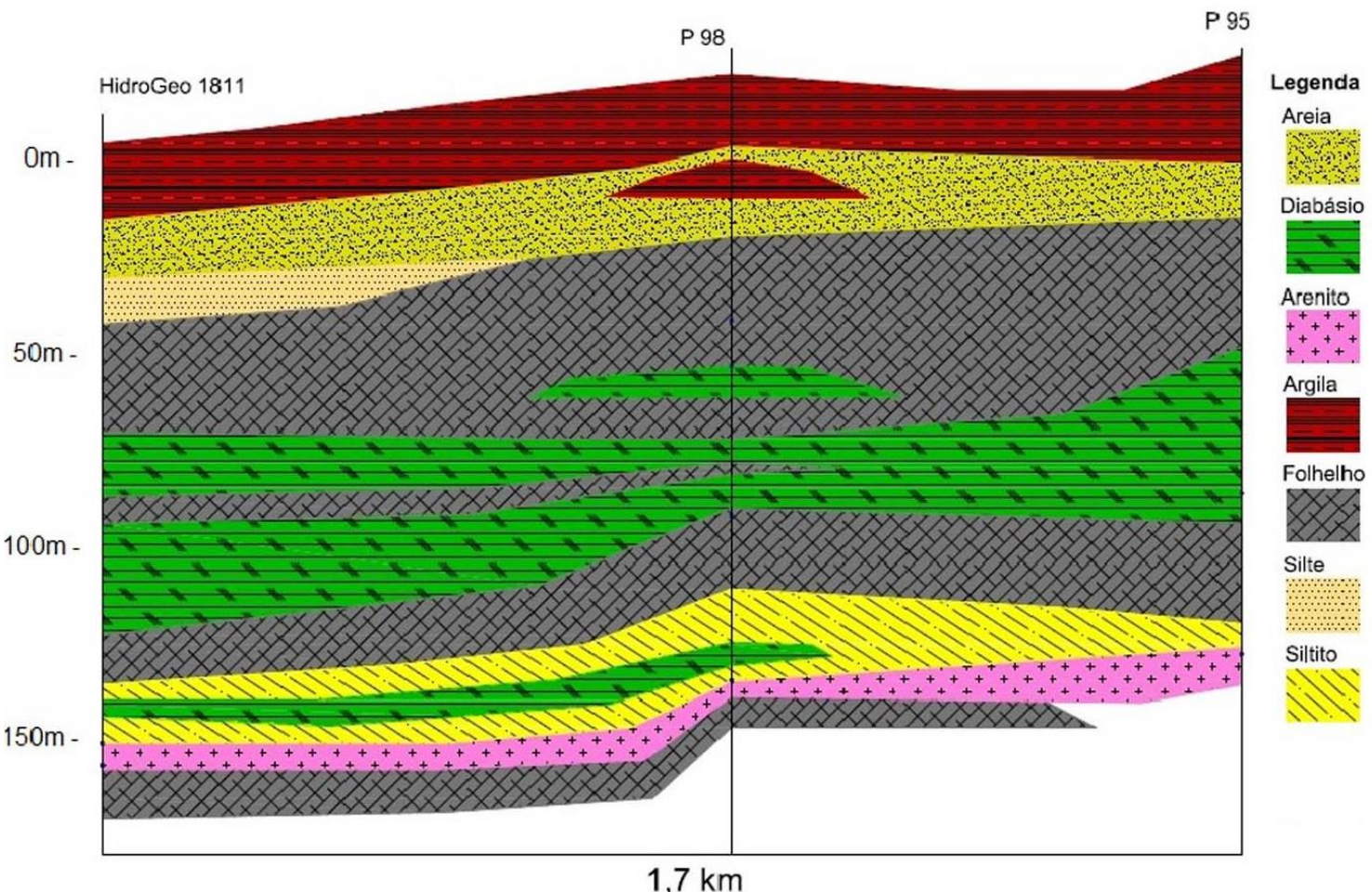

Figura 6 - Seção Geológica 1

Figure 6 - Geological Sections 1 


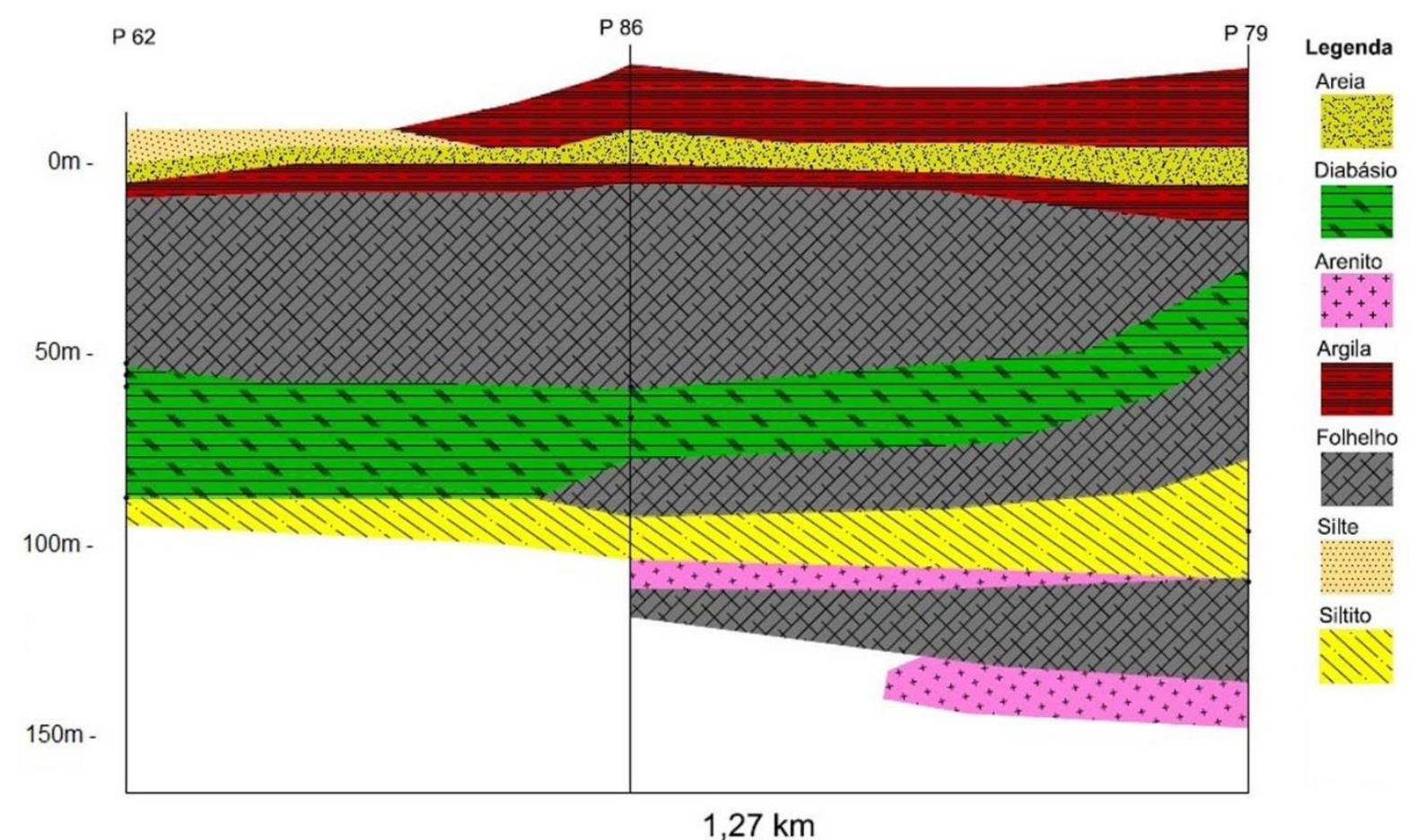

Figura 7 - Seção Geológica 2

Figure 7 - Geological Sections 2

Analisando os dados geológicos e as seções geradas, observa-se que as camadas mais superficiais são representadas por depósitos sedimentares (argilas, areias e siltes) típicos da Formação Rio do Rastro. As camadas inferiores estão representadas por folhelhos (Formação Irati e Estrada Nova), siltitos (Formação Palermo) e folhelhos escuros e arenitos (Formação Rio Bonito). As camadas de folhelho possuem espessuras variáveis entre 6 e $50 \mathrm{~m}$ e podem ser encontradas em profundidades de 30 até $175 \mathrm{~m}$. As camadas de arenito possuem espessuras médias de $8,2 \mathrm{~m}$ e são encontradas em profundidades médias de 155,5 m. Além disso, há ocorrência de intrusões de diabásio que cortam as rochas sedimentares em diferentes locais e profundidades. Essas intrusões deram origem a fraturas que cortam as diferentes litologias.

Os aquíferos identificados na região estão associados às fraturas existentes nos folhelhos, siltitos e diabásio e as camadas de arenitos, sendo que os folhelhos e arenitos correspondem aos principais aquíferos captados pelos poços tubulares. Dessa forma, os poços não captam água dos aquíferos que formam o Sistema Aquífero Quaternário Costeiro II. Deve-se destacar que um poço pode captar água das fraturas associadas aos folhelhos, bem como dos arenitos que se encontram em maior profundidade. A profundidade dos poços varia entre $62,5 \mathrm{~m}$ a $243 \mathrm{~m}$, com uma média de $130 \mathrm{~m}$.

A vulnerabilidade natural dos aquíferos foi avaliada através da metodologia GOD, sendo que para cada um dos parâmetros foi gerado um mapa temático. Na Tabela 2 são apresentados os valores que foram definidos para cada um dos parâmetros $\mathrm{G}, \mathrm{O}$ e $\mathrm{D}$, bem como os valores resultantes do cálculo da vulnerabilidade (multiplicação dos parâmetros $\mathrm{G} \times \mathrm{O}$ x D).

$O$ parâmetro $G$ foi definido com base na interpretação dos perfis geológicos sendo que os valores selecionados variaram entre 0,2 (aquífero associado a camadas de arenito que está localizado a uma maior profundidade e possui camadas confinando o mesmo) e 0,6 (aquíferos associados aos folhelhos, diabásios e siltitos). A interpolação desses valores resultou no mapa temático do parâmetro G, apresentado na Figura 8.

A análise dos perfis geológicos dos poços indicou que os principais estratos de cobertura correspondem a argilas, siltes e areias que foram utilizados na definição dos valores do parâmetro O. Os valores definidos variaram entre 0,4 (camadas de argila), 0,5 (camadas de siltes), 0,6 (camadas de folhelhos) e 0,7 (areias aluviais). Com base nesses dados foi gerado o mapa temático do parâmetro $O$ (Figura 9).

$\mathrm{O}$ parâmetro $\mathrm{D}$ foi definido com base na avaliação dos níveis de água dos poços, sendo que os valores selecionados variaram entre 0,6 (mais de $50 \mathrm{~m}$ de profundidade) e 0,9 (até $5 \mathrm{~m}$ ). Na Figura 10 é apresentado o mapa temático do parâmetro $\mathrm{D}$. 
A partir dos 3 mapas foi feita a multiplicação dos valores de cada mapa e gerado o mapa de vulnerabilidade natural dos aquíferos que é apresentado na Figura 11.

Para a área de estudo foram definidas 3 classes de vulnerabilidade: insignificante, baixa e média. A vulnerabilidade predominante na área de estudo foi a baixa, cobrindo $94,12 \%$ da região, seguida pela média com $3,08 \%$ de área e por último a insignigicante, que corresponde a $2,80 \%$ da área.

Tabela 2 - Parâmetros G, O, D

Table 2 - G,O,D parameters

\begin{tabular}{|c|c|c|c|c|c|}
\hline $\begin{array}{c}\text { Identificação- № } \\
\text { Poço }\end{array}$ & Parâmetro G & Parâmetro $\mathrm{O}$ & Parâmetro D & G.O.D & Primeiro aquífero captado \\
\hline P008 & 0,4 & 0,4 & 0,8 & 0,128 & Folhelho \\
\hline P009 & 0,3 & 0,7 & 0,8 & 0,168 & Arenito Fino \\
\hline P010 & 0,4 & 0,5 & 0,7 & 0,140 & Diabásio \\
\hline P011 & 0,6 & 0,5 & 0,7 & 0,210 & - \\
\hline P012 & 0,6 & 0,4 & 0,7 & 0,168 & - \\
\hline P015 & 0,6 & 0,5 & 0,8 & 0,240 & - \\
\hline P016 & 0,6 & 0,5 & 0,7 & 0,210 & - \\
\hline P017 & 0,6 & 0,7 & 0,7 & 0,294 & - \\
\hline P018 & 0,6 & 0,7 & 0,8 & 0,336 & - \\
\hline P019 & 0,6 & 0,7 & 0,9 & 0,378 & - \\
\hline P020 & 0,6 & 0,7 & 0,8 & 0,336 & - \\
\hline P026 & 0,6 & 0,6 & 0,7 & 0,252 & - \\
\hline P027 & 0,5 & 0,5 & 0,7 & 0,175 & Folhelho \\
\hline P028 & 0,6 & 0,6 & 0,8 & 0,288 & - \\
\hline P030 & 0,5 & 0,4 & 0,6 & 0,120 & Folhelho \\
\hline P033 & 0,6 & 0,7 & 0,8 & 0,336 & - \\
\hline P035 & 0,6 & 0,7 & 0,8 & 0,336 & Folhelho \\
\hline P036 & 0,5 & 0,4 & 0,8 & 0,160 & Quartzito \\
\hline P038 & 0,6 & 0,4 & 0,7 & 0,168 & Folhelho \\
\hline P039 & 0,6 & 0,4 & 0,8 & 0,192 & Folhelho \\
\hline P040 & 0,6 & 0,7 & 0,7 & 0,294 & Folhelho \\
\hline P044 & 0,6 & 0,6 & 0,7 & 0,252 & Folhelho \\
\hline P047 & 0,5 & 0,4 & 0,8 & 0,160 & Diabásio \\
\hline P052 & 0,5 & 0,7 & 0,7 & 0,245 & Diabásio \\
\hline P054 & 0,5 & 0,4 & 0,8 & 0,160 & Folhelho \\
\hline P055 & 0,6 & 0,7 & 0,7 & 0,294 & Folhelho \\
\hline P062 & 0,5 & 0,5 & 0,8 & 0,200 & Diabásio \\
\hline P063 & 0,5 & 0,4 & 0,7 & 0,140 & Diabásio \\
\hline P073 & 0,6 & 0,4 & 0,9 & 0,216 & Folhelho \\
\hline P076 & 0,6 & 0,7 & 0,7 & 0,294 & - \\
\hline P078 & 0,6 & 0,4 & 0,7 & 0,168 & Diabásio \\
\hline P079 & 0,4 & 0,7 & 0,6 & 0,168 & Siltito \\
\hline P080 & 0,6 & 0,4 & 0,7 & 0,168 & Folhelho \\
\hline P081 & 0,6 & 0,7 & 0,7 & 0,294 & - \\
\hline P086 & 0,6 & 0,4 & 0,7 & 0,168 & Folhelho \\
\hline P088 & 0,6 & 0,4 & 0,7 & 0,168 & Folhelho \\
\hline P090 & 0,6 & 0,4 & 0,8 & 0,192 & Folhelho \\
\hline P091 & 0,2 & 0,4 & 0,7 & 0,056 & Arenito Fino \\
\hline P092 & 0,2 & 0,4 & 0,6 & 0,048 & Arenito Médio \\
\hline P095 & 0,5 & 0,4 & 0,7 & 0,140 & Diabásio \\
\hline P097 & 0,6 & 0,4 & 0,9 & 0,216 & - \\
\hline P098 & 0,6 & 0,4 & 0,6 & 0,144 & Folhelho \\
\hline P100 & 0,5 & 0,4 & 0,7 & 0,140 & Folhelho \\
\hline P102 & 0,4 & 0,4 & 0,7 & 0,112 & Diabásio \\
\hline P1811 & 0,2 & 0,4 & 0,7 & 0,056 & Arenito Fino \\
\hline P2380 & 0,6 & 0,4 & 0,8 & 0,192 & Folhelho \\
\hline
\end{tabular}




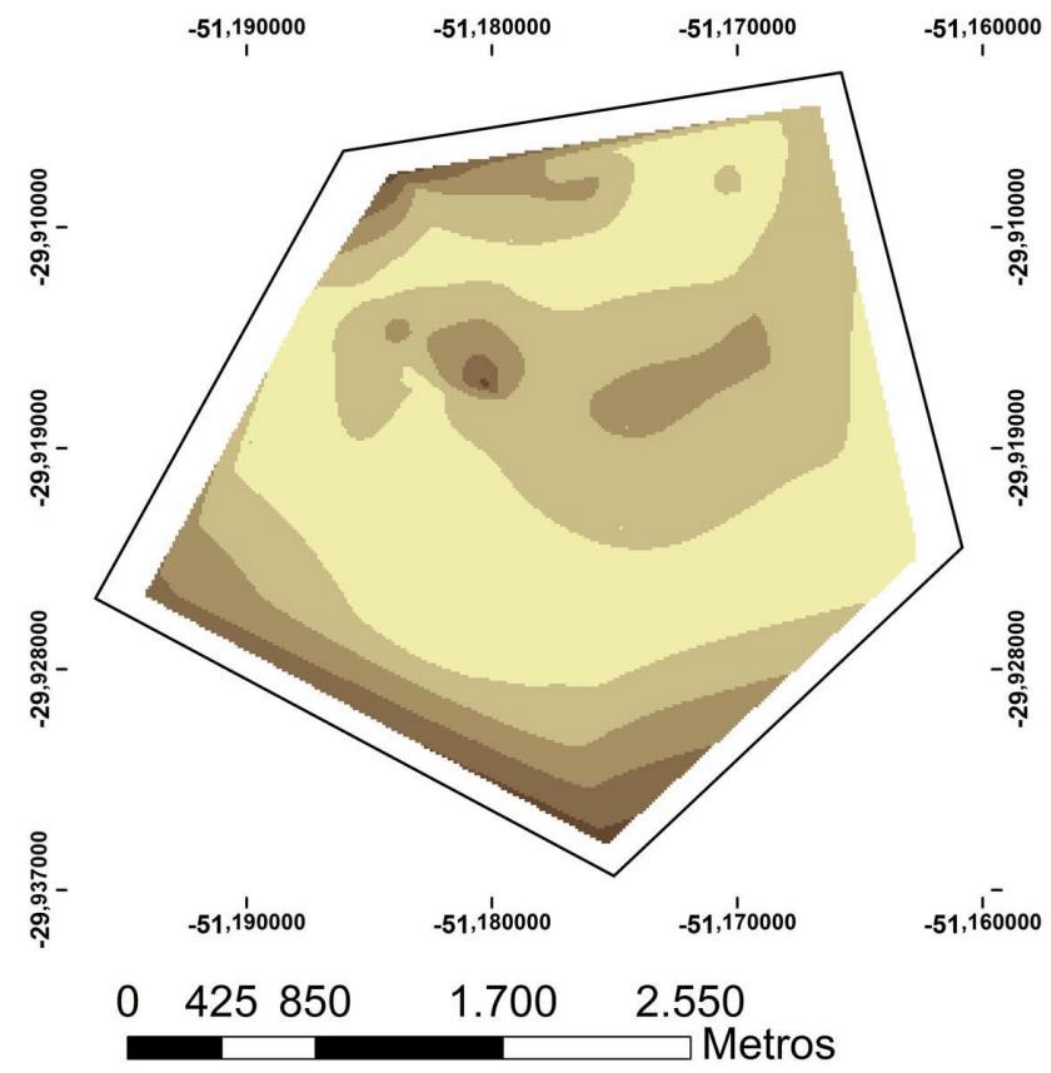

Mapa temático do Parâmetro G

Parâmetro G - Grau de Confinamento da água subterrãnea

Escala 1:24.000

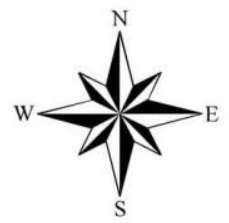

Figura 8 - Mapa temático do parâmetro G

Figure 8 - Thematic map of G parameter

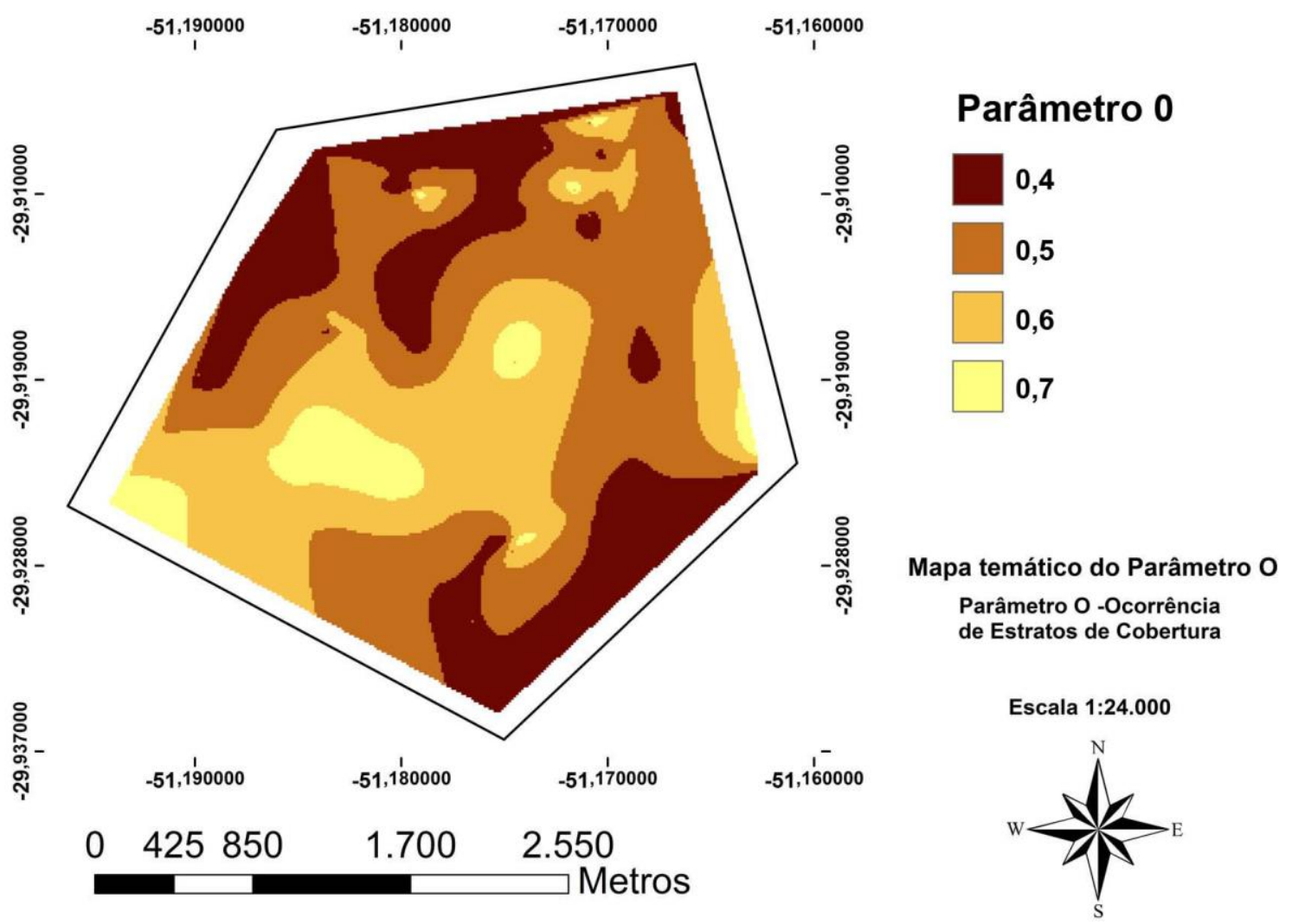

Figura 9 - Mapa temático do parâmetro O

Figure 9 - Thematic map of $\mathrm{O}$ parameter 


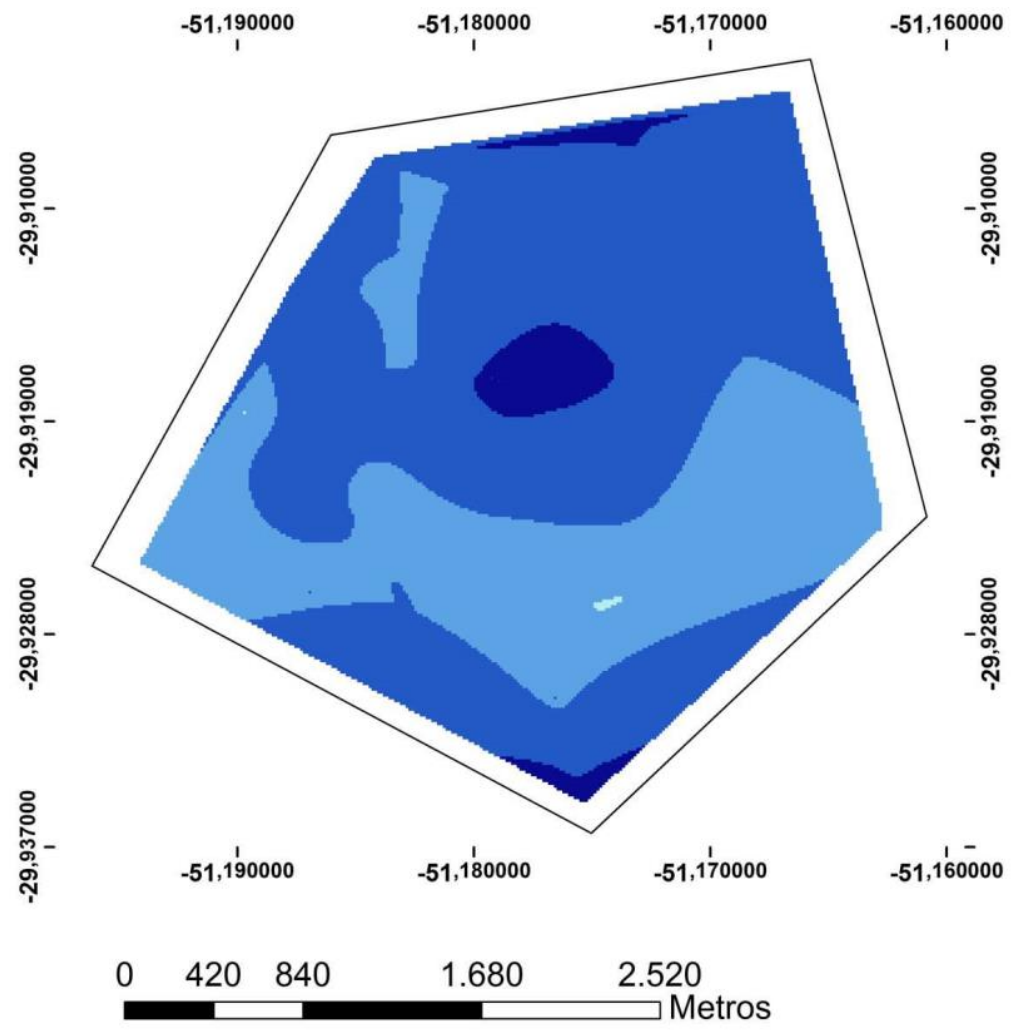

\section{Parâmetro D}

$>50 m-0,6$

$20 m$ a $50 m-0,7$

5 a $20 m-0,8$

$<5 m-0,9$

Mapa temático do Parâmetro D

Parâmetro D - Profundidade de aquiferos Escala 1: 24.000

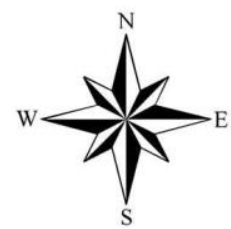

Figura 10 - Mapa temático do parâmetro D

Figure 10 - Thematic map of D parameter

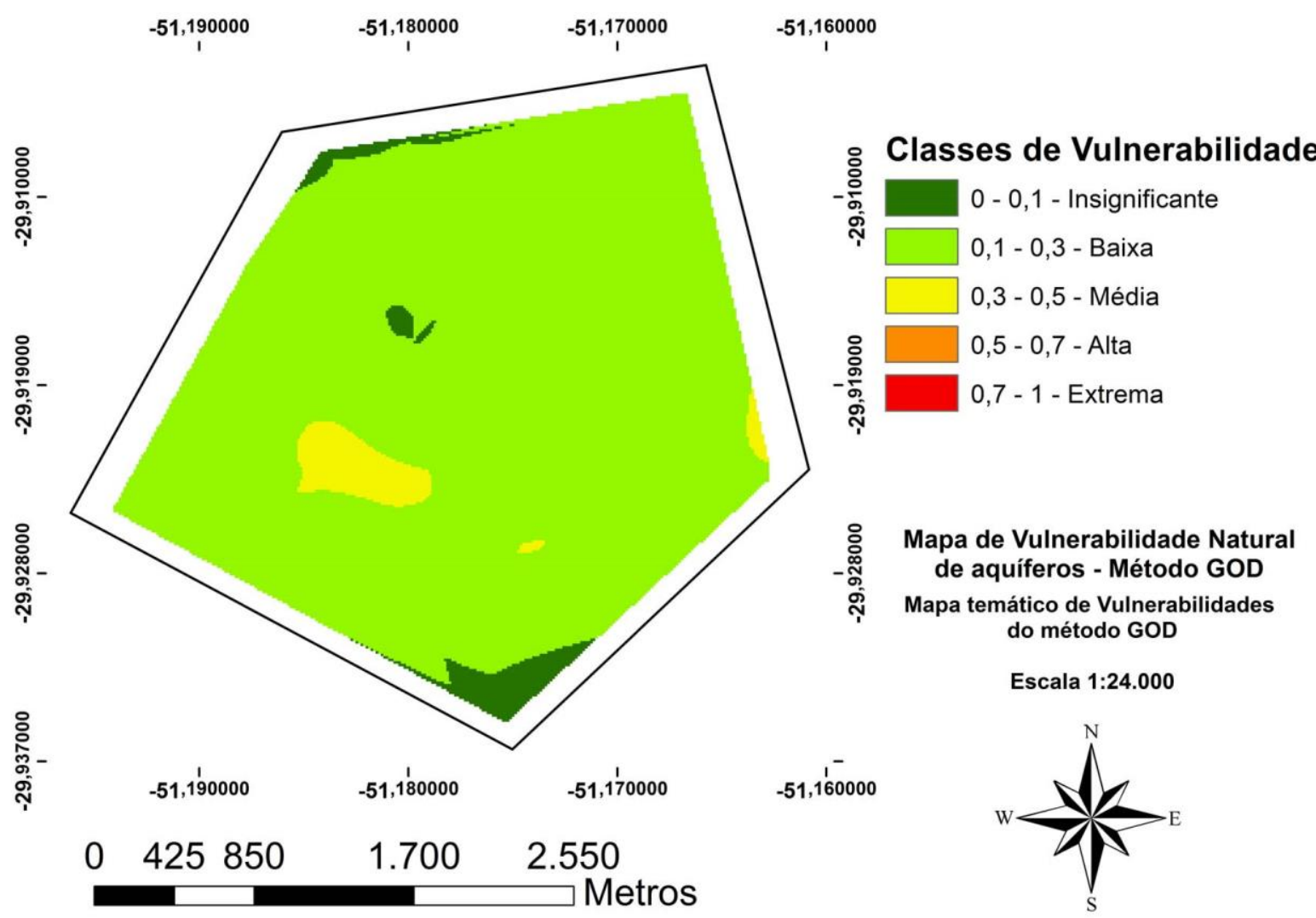

Figura 11 - Mapa temático de vulnerabilidade Natural de aquíferos

Figure 11 - Thematic map of aquifer natural vulnerability 
A identificação de uma predominância de vulnerabilidade baixa para a região de estudo é explicada, pois os aquíferos estão localizados a uma maior profundidade, sendo que em geral, possuem uma cobertura de sedimentos de granulação fina (argilas e siltes). Aliado a isso, a profundidade dos níveis de água é, na sua maioria, superior a 20 metros. Esses dados indicam que a área apresenta uma baixa acessibilidade hidráulica em função das características geológicas e hidrogeológicas e uma baixa vulnerabilidade natural a contaminação. Nas áreas onde há ocorrência de aquíferos fraturados que estão recobertos por depósitos de sedimentos arenosos e que possuem níveis de água pouco profundos (inferiores a 20 metros) a vulnerabilidade passa a ser média. Essas áreas, apresentam um maior potencial a contaminação pois a acessibilidade hidráulica é aumentada em função das características geológicas e hidrogeológicas.

Porém, mesmo que na área tenha sido identificado um predomínio de vulnerabilidade baixa a insignificante $(96,92 \%)$, os aquíferos podem ser contaminados. A vulnerabilidade natural do aquífero não leva em consideração as contaminações geradas através de poços mal construídos ou que são explotados sem controle. Poços que apresentam problemas construtivos tornam-se vias de contaminação do aquífero, pondo em risco os usuários da água subterrânea.

\section{CONCLUSÕES}

$\mathrm{Na}$ área de estudo há ocorrência de dois aquíferos principais, os fraturados e os granulares confinados. Os aquíferos fraturados estão associados a estruturas presentes em folhelhos e siltitos, que foram originadas pelas intrusões de diabásio que cortaram essas rochas. Esses aquíferos ocorrem em diferentes profundidades sendo que os poços que captam os mesmos podem apresentar mais de uma entrada de água. Os aquíferos granu-

\section{REFERÊNCIAS}

ALLER, L.; BENNET, T.; LEHR, J.H.; PETTY, R.; HACKETT, G. DRASTIC: a standardized system for evaluating grondwater pollution potential using hydrogeologyc settings. US E.P.A. - EPA/600/2-87/035, 1987.

ANA (BRASIL). Atlas Brasil: abastecimento urbano de água: panorama nacional. Agência Nacional de Águas; Engecorps/Cobrape - Brasília : ANA : Engecorpos/ Cobrape, 2010. Volume 1 e 2.

CPRM. Carta Geológica do Estado do Rio Grande do Sul, Porto Alegre. Ministério das Minas e Energia. 2008 . lares estão associados a arenitos e ocorrem em profundidades maiores, abaixo das camadas de siltitos, folhelhos e diabásios. Em função disso, esses aquíferos podem ser considerados como granulares confinados.

As camadas mais superficiais que recobrem esses aquíferos consistem de depósitos sedimentares argilosos, siltosos e arenosos, sendo que os primeiros predominam na região.

Os poços tubulares identificados na área captam água tanto dos aquíferos fraturados como dos granulares confinados, sendo utilizados principalmente, por condomínios residenciais.

A interpretação dos dados geológicos e hidrogeológicos permitiu definir diferentes pesos para os parâmetros G, O e D (metodologia GOD). Os valores do parâmetro $G$ variaram entre 0,2 (confinado) e 0,6 (não confinado coberto), enquanto que para o parâmetro $\mathrm{O}$ foram definidos valores entre 0,4 (argilas) e 0,7 (areias aluviais). Por fim, o parâmetro D apresentou valores entre 0,6 e 0,9 visto que as profundidades dos níveis de água são variáveis, mas na sua maioria superiores a 20 metros.

Com base nesses valores foi determinada a vulnerabilidade natural dos aquíferos, sendo que na maior parte da área a mesma foi considerada como baixa a insignificante. Isso é explicado, pois na região predominam aquíferos localizados em numa maior profundidade que são recobertos por camadas de sedimentos finos (argilas e siltes) e a profundidade dos níveis de água, na sua maioria, é superior a 20 metros.

No entanto, a baixa vulnerabilidade encontrada não exclui a possibilidade de ocorrência de contaminação, pois poços tubulares que captam água dos aquíferos fraturados associados ao folhelho, que possuem entradas de água mais próximas a superfície e que são recobertos por sedimentos arenosos ou siltosos, podem se tornar mais vulneráveis.

ESRI, Software ArcGis, Versão 10.0. Redlands, CA: Environmental Systems Research Institute, 2010.

FAMURS. Dados Gerais - Canoas/RS. Disponível em: <http://www.portalmunicipal.org.br/entidades/famurs/da do_geral/mumain.asp?ilildE=5523\&ildMun $=100143074$ > último acesso em 23/05/2013.

FOSTER, S. S. D.; HIRATA, R. C. A. Groundwater pollution risk evaluation: the methodology using available data. Lima: CEPIS/PAHO/WHO, 1988.

FOSTER, S.; HIRATA, R.; GOMES, D.; D'ELIA, M.; PARIS, M. Proteção da qualidade da água subterrâ- 
nea: um guia para empresas de abastecimento de água, órgãos municipais e agências ambientais. Banco Internacional de Reconstrução e Desenvolvimento/Banco Mundial, 2006.

IBGE. Censo Demográfico 2010. IBGE, abril de 2011. Disponível em <http://censo2010.ibge.gov.br/pt/>. Acesso em 24/04/2013.

MACHADO, J. L.; FREITAS, M. A. de. Projeto Mapa Hidrogeológico do Rio Grande do Sul: Escala 1:750.000, relatório final. Porto Alegre: CPRM, 2005. 1 CD-ROM.

MARQUEZAN, R. G. Análise de recursos digitais como ferramentas de avaliação em ações de proteção de aquíferos no trajeto de oleodutos. 2008. 184p. Tese (Doutorado) - Instituto de Pesquisas Hi- dráulicas, Universidade Federal do Rio Grande do Sul, Porto Alegre, 2008.

MEDEIROS, C. M.; RIBEIRO, M. A. de F. M.; RUFINO, I. A. A.; BARBOSA, D. L.; Mapeamento da vulnerabilidade de parte da Bacia Sedimentar do Baixo Curso do Rio Paraíba utilizando o método GOD. In: SIMPÓSIO BRASILEIRO DE SENSORIAMENTO REMOTO SBSR, 15., Curitiba, PR, 2011. Anais, INPE, p. 3819.

SIAGAS. Portal SIAGAS. Disponível em: <http://siagasweb.cprm.gov.br/layout/>. Acesso em: 14/04/2013.

VIERO, A. C. Geodiversidade do Estado do Rio Grande do Sul. Porto Alegre: CPRM, 2010. 250p; $30 \mathrm{~cm}, 1$ DVD-ROM. 